\title{
Variability of Platanus orientalis leaves under a heterogeneous environment in the Hissar Valley of Tajikistan
}

\author{
Saifiddin Davlatzoda ${ }^{1^{*}}$, Jamoliddin Bobokalonov ${ }^{2}$, and Elena Baikova ${ }^{3,4}$ \\ ${ }^{1}$ Bohtar State University named after Nosir Khusrav, 735140 Kurgan-Tyube, Tajikistan \\ ${ }^{2}$ Tajik National University, 734025 Dushanbe, Tajikistan \\ ${ }^{3}$ Central Siberian Botanical Garden of the Siberian Branch of the Russian Academy of Sciences \\ (CSBG SB RAS), 630090 Novosibirsk, Russia \\ ${ }^{4}$ Novosibirsk State University, 630090 Novosibirsk, Russia
}

\begin{abstract}
Geospatial monitoring of leaves variability has been performed for Platanus orientalis L. (Platanaceae) in Hissar Valley of Tajikistan. 17 local populations were clustered in four territorial groups and investigated by 11 morphometric variables and four indices. Six levels of variability were taken in the classification of S.A. Mamaev. All tested parameters were ranked due to the total score of variability levels. It was established, that indicators which have the combination of high, increased, and medium levels of variability, can be recommended for the purpose of geospatial monitoring. In the studied set of variables are two such ones: (1) the ratio of the length of leaf blade to the lengh of petiole, and (2) the petiole lengh. These two indicators reliably respond to point sources of industrial environmental pollution (Aluminum and Cement factories). Six indicators with domination of the middle level of variability, are of diagnostic value to differ loci inside territorial groups of populations. The results of the experiment confirmed, that set of variability levels for 8 of 15 studied parameters has diagnostic matter for geospatial monitoring of environment conditions in Hissar Valley of Tajikistan.
\end{abstract}

\section{Introduction}

Geospatial monitoring of environment in densely populated areas of the Earth'a surface, including inter-mountain valleys, is highly urgent scientific problem, requiring a system analysis in situation of increasing people life expectancy. To get this purpose, it is necessary to do regional tests with focusing on details of the modern economic development. Platanus orientalis L. (Platanaceae) is wide-spread species of woody plants, distributed from Middle Sea territory and further up to Middle Asia. This species has been introduced into the culture from ancient times and is used in landscaping. $P$. orientalis is characterized by high level of ecological mobility and resistence against heavy metals pollution in megapolices [1].

*Corresponding author: s.davlatov@mail.ru 
The purpose of the scientific experiments is to investigate prospects of environmemt monitoring in Hissar Valley of Tajikistan on the base of the diversity of variability levels of leaves for P. orientalis.

\section{Material and methods}

Representative sets of mature leaves named as an experimental leaves series were taken to calculate vabiability levels in leaf morphological micrometry of Platanus orientalis. The series were collected in all available parts of three separately growing trees in the generative phase of development. Morphometric method and statistical approaches were used in the study applied. Experimental leaves series were collected on reperentative sites named here as localitets, or locations, to get ecoinformation from all the parts of area of $P$. orientalis in the Hissar Valley of Tajikistan. So, we have natural locations with different anthropogenous press, caused point sources of industrial pollution (Alimunum Plant, Cement Plant, Thermal Power Station) and linear pollution sources of highways. Experimental materials were selected in 17 localitets in 2012 and 2013, and three additional ones in 2013. Local populations of the species were chosen in all the parts of Hissar Valley, and form four territorial clusters, listed here in West to East direction: (1) Tursunzadecluster, including Shahrinav locations (sites №№7-12); (2) Hissar-cluster (sites №№1315); (3) Dushanbe-cluster (sites №№1-6), and (4) Vakhdat-cluster (sites №№16,17). Tursunzade and Hissar territorial clusters are together the western group of sites, and Dushanbe and Vakhdat clusters we include in the eastern group of sites. There are the first point of industrial pollution - Aluminum Plant, doing negative influence on the sites 1.1, 1.2 and 1.3 in Tursunzade cluster; and the second one, Cement Plant, doing negative influence on the sites 3.1, 3.2, and 3.3 in Dushanbe cluster. Thermal Power Station is on the site 3.3, and its influenses nagatively on this location. Representative sites of Hissar and Vakhdat clusters are nor directly affected by spurces of industrial pollution and can be taken into account as control sites having only native processes, since they are out of anthropogenous press.

Each leaf in the series was metered of 19 parameters (or indicalors), in this count 16 create pairs. Thus, 11 indicators are (Figure 1): 1) ab - the petiole lengh; 2) bc - the distance from petiole top to base of lateral vein of the first order; 3) bd - the leaf blade lengh; 4) ce, cf - the distance from the base of lateral primary vein to the base of lateral secondary vein on the central vein of the lower lobes; 5) ei, fj - the lengh of central veins on the lower lobes; 6) $\mathrm{cg}$, ch - the lengh of central veins on the middle lobes; 7) $\mathrm{ko}, \mathrm{lp}$ - the distance from the sinus between the lower and the middle lobes up to the central vein; 8) $\mathrm{mq}, \mathrm{nr}$ - the distance from the sinus between the middle and central lobes up to the central vein; 9) es, $\mathrm{ft}$ - the distance from the base of the central vein on the lower lobes to the central vein of the blade; 10) eco, fco - the angles of departure of the median veins of the middle lobes from the main vein; 11) ieg, jfh - the angles of depature of the median veins of the lower lobes from the central veins of the middle lobes. Additionally, four indices were calculated: (1) leaf index (the ratio of leaf blade length to petiole length), (2) leaf blade index (the ratio of leaf blade length to the distance between sinuses of the lower and middle lobes), and two indices of the shape for the leaf blade: (3) the ratio of the distance from the point of departure of the lateral veins of the middle lobes to the length of the central vein, (4) the ratio of the distance between sinuses of the lower and middle lobes to the distance between sinuses of the middle and central (apical) lobes. 


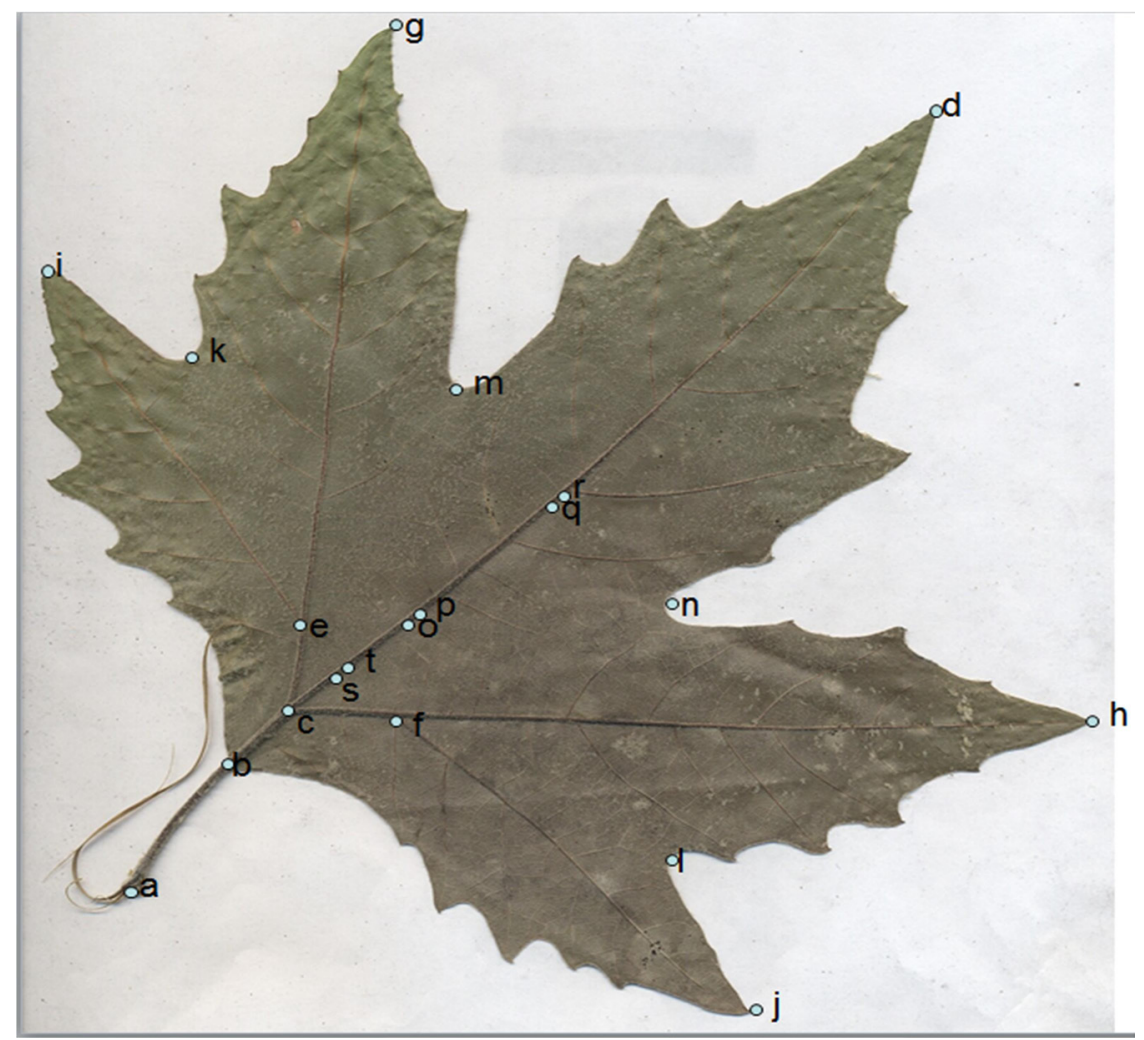

Fig. 1. Morphometric indicators used for assessment of variability in leaf morphology of Platanus orientalis.

The variability of the morphometric parameters of the Platanus orientalis leaves and additionally calculated indices was assessed on the S.A. Mamaev scale [2], specially designed to study the variability of morphological characters of woody plants. This scale includes six levels: level 1 (very low) - the coefficient of variation has values less than $7.0 \%$; level 2 (low) - 7.1-12.0\%; level 3 (medium) - 12.1-20.0\%; level 4 (increased) 20.1-30.0\%; level 5 (high) - 30.1-40.0\%; level 6 (very high) - more than $40.0 \%$. The coefficient of variation is a measure of the relative dispersion of values of the measured parameter; it shows what the part of the average value of this parameter is its average dispersion.

\section{Results and Discussion}

In accordance with the adopted scale, variability levels for morphometric parameters and indices were calculated in all experimental sets (leaves series) for each locality of $P$. orientalis in Hissar Valley of Tajikistan. Obtained results with geospatial diversity in variability levels for morphometric parameters and indices are presented in Table 1.

All tested parameters were ranked due to the decreasing total score of variability levels as the sum of levels in all the set of local populations. Score for each leaf parameter in the locality we do equal to variability level number after Mamaev's scale [1]. For example, the most variative parameter (pair of ce, $\mathrm{cf}$ - the distance from the base of lateral vein of the first order to the base of the lateral vein of the second order on the central vein of the lower lobe) gets 98 points, having 6th variability level in 13 localities, and 5th level in 4 rest ones. 
In accordance with results obtained, only three of 15 parameters have 6th variability level. Two other of them are: (1) the distance from the base of the central vein of the lower lobes to the central vein of a blade (pair es, ft; 97 points), and (2) the distance from the petiole top to the base of the lateral vein of the first order (bc; 83 points). Parameters ce, $\mathrm{cf}$ and es, $\mathrm{ft}$ have maximum geospatial similarity in variability levels and the same in 16 of 17 locations. The difference was revealed in location 1.2 only, where parameter es, ft has 4th variability level, one step below than the parameter ce, cf. High degree of similarity in geospatial patterns of variability levels for these two parameters marks success in using methods of morphogenetic coordinarion and correlative analysis in this pair [2].

Table 1. Geospatial diversity in variability levels of morphometric parameters and indices of Platanus orientalis leaves.

\begin{tabular}{|c|c|c|c|c|c|c|c|c|c|c|c|c|c|c|c|c|c|c|}
\hline Localities: & 1.1 & 1.2 & 1.3 & 1.4 & 1.5 & 1.6 & 2.1 & 2.2 & 2.3 & 3.1 & 3.2 & 3.3 & 3.4 & 3.5 & 3.6 & 4.1 & 4.2 & \\
\hline Parameters: & 6 & 5 & 6 & 5 & 6 & 6 & 6 & 6 & 6 & 6 & 6 & 6 & 6 & 6 & 6 & 5 & 5 & \begin{tabular}{|c} 
Sumar \\
score: \\
98
\end{tabular} \\
\hline es, $\mathrm{ft}$ & 6 & 4 & 6 & 5 & 6 & 6 & 6 & 6 & 6 & 6 & 6 & 6 & 6 & 6 & 6 & 5 & 5 & 97 \\
\hline $\mathrm{bc}$ & 6 & 4 & 6 & 6 & 6 & 6 & 6 & 6 & 6 & 3 & 4 & 3 & 4 & 3 & 6 & 4 & 4 & 83 \\
\hline leaf index & 5 & 4 & 3 & 3 & 4 & 4 & 4 & 4 & 5 & 3 & 3 & 5 & 4 & 5 & 4 & 5 & 4 & 69 \\
\hline $\mathrm{ab}$ & 5 & 4 & 2 & 3 & 4 & 5 & 4 & 3 & 5 & 3 & 3 & 5 & 3 & 5 & 4 & 5 & 4 & 67 \\
\hline ei, fj & 3 & 4 & 3 & 3 & 5 & 5 & 3 & 3 & 4 & 3 & 3 & 3 & 3 & 4 & 3 & 3 & 4 & 59 \\
\hline $\mathrm{mq}, \mathrm{nr}$ & 3 & 3 & 3 & 3 & 4 & 4 & 3 & 3 & 3 & 3 & 3 & 3 & 4 & 2 & 4 & 3 & 3 & 54 \\
\hline eco, fco & 3 & 3 & 3 & 3 & 3 & 3 & 3 & 4 & 2 & 2 & 3 & 3 & 2 & 3 & 3 & 3 & 4 & 50 \\
\hline ko, lp & 3 & 3 & 3 & 3 & 3 & 4 & 2 & 3 & 3 & 3 & 3 & 3 & 3 & 2 & 3 & 3 & 3 & 50 \\
\hline ieg, gfh & 3 & 3 & 2 & 3 & 4 & 3 & 3 & 2 & 3 & 3 & 2 & 3 & 2 & 3 & 3 & 3 & 3 & 48 \\
\hline $\mathrm{cg}, \mathrm{ch}$ & 3 & 3 & 2 & 3 & 3 & 3 & 2 & 3 & 3 & 2 & 2 & 2 & 3 & 3 & 3 & 3 & 4 & 47 \\
\hline bd & 3 & 3 & 2 & 2 & 2 & 3 & 2 & 2 & 2 & 2 & 2 & 3 & 2 & 2 & 3 & 3 & 3 & 41 \\
\hline blade index & 3 & 3 & 2 & 1 & 4 & 3 & 2 & 1 & 3 & 1 & 1 & 3 & 2 & 2 & 2 & 2 & 2 & 37 \\
\hline form index 2 & 2 & 2 & 2 & 2 & 3 & 3 & 2 & 1 & 2 & 1 & 2 & 2 & 2 & 1 & 2 & 3 & 3 & 35 \\
\hline form index 1 & 1 & 1 & 1 & 1 & 1 & 1 & 1 & 1 & 1 & 1 & 1 & 1 & 1 & 1 & 1 & 1 & 1 & 17 \\
\hline
\end{tabular}

Parameter bc has the total score of variability levels 83 points, i.e. 14-15 points lower than of parameters ce, $\mathrm{cf}$ and es, $\mathrm{ft}$. The distance from the top of the petiole top to the base of the lateral vein of the first order demonstrates significant changes of variability levels, the most contract (three steps) in the Dushanbe territorial group, between 3.5 and 3.6 locations. This indicator can be recommended to evaluate differency level between western and eastern groups of local populations, because its variability levels is changed contrastly in the tranzit zone between Hissar to Dushanbe groups in the central part of Hissar Valley. This indicator can be helpful within investigations of coordanations in hereditary and nonhereditary variability, as well as modification variability $[3,4]$ in local populations of $P$. orientalis for Hissar Valley in total scale.

Further, in the order of value desceasing of the sumar score of variability levels, we get: the ratio of the length of leaf blade to the lengh of petiole, named leaf index (69 points), and parameter $\mathrm{ab}$ - the petiole lengh (67 points). They are characterized by combination of high, increased and medium levels of variability as in the western groups and in the eastern territorial groups and have good effect to do geospatial monitoring to assess environmental 
influence on the variavility levels of leaves of $P$. orientalis. These two indicators reliably respond to point sources of industrial environmental pollution (Aluminum and Cement plants). In the series of localitets 1.1-1.2-1.3 of Tursunzade-claster we can see the zone of Aluminum plant industrial influence, and variability level is down consistently from 5 to 3 , with $4^{\text {th }}$ level on the control sites $(1.5$ and 1.6). In the same series, variability level of the lengh of petiole shuts down even more, from 5 to 2 (see Table 1, indicator ab). Hence, indicators which have the combination of high, increased, and medium levels of variability, have the main significance in determination of industrial pollution degree for environment in the Hissar Valley of Tajikistan.

Six indicators have the sumar score of 59 to 47 points and are characterized by predominance of a 3 th (middle) level in the most sites. An increased level of variability in this group of indicators is find rarely. High level is detected only twice - for indicator ei, $\mathrm{fj}$ in Tursunzade-cluster at control sites 1.5 and 1.6, and this fact indicates its prospects in diagnosing the industrial pollution degree for the environment in the Hissar Valley of Tajikistan. In this group of indicators the low level occurs in rare case, and can be recommended as diagnostic for sites with accumulate effect of point industrial pollution (for example, indicators ieg, gfh and $\mathrm{cg}$, ch for site 1.3). Hence, indicators with domination of the middle level of variability, are of diagnostic value to differ sites inside territorial clusters. The results of the experiment confirmed, that set of variability levels for 8 of 15 studied parameters has diagnostic matter for geospatial monitoring of environment conditions in Hissar Valley of Tajikistan.

\section{References}

1. E. Khosropour, P. Attarod, A. Shirvany, et al., J. For. Res., 30, 1437-1445 (2019)

2. S.A. Mamaev, Forms of intraspecific variability of woody plants (on the example of the Pinaceae family in the Urals) (Publishing House Nauka, Moscow, 1973)

3. N.S. Rostova, Correlations: structure and variability (Publishing House of St. Petersburg University, St. Petersburg, 2002)

4. S.G. Inge-Vechtomov, Ecological Genetics, 8, 4-9 (2010)

5. O.N. Tikhodeev, Ecological Genetics, 10, 56-65 (2012) 